\title{
Do environmental factors moderate gender differences in cognition across generations?
}

\author{
A. Vardiampasis ${ }^{1}$, C. Gramandani ${ }^{2}$. \\ ${ }^{1}$ General Hospital Of Rethymnon, Mental Health Center of Rethymnon, Crete Greece. \\ ${ }^{2}$ General Hospital of Rethymnon, Mental Health Center of Rethymnon, Crete, Greece.
}

\section{Objectives:}

The purpose of this study was to examine if brain changes across generations due to environmental factors, and whether gender differences in cognitive abilities are moderated.

Background:

Digit ratio, especially 2D:4D ratio, is a potential proxy marker for prenatal androgen exposure, which has been reported to be associated with cognitive abilities. There is strong evidence suggesting that there are differences between genders in digit ratio and cognition.

Materials and Methods:

93 women and 89 men, have been divided into three age groups: 20-30 y.0. (31 women-32 men), 40-50 y.0. (32 women-29 men), and 70-80 y.o. (30 women-28 men). The sample consisted of people with similar educational level $(>9$ y.0.), MMSE score $>\mathbf{2 5}$, and Montreal Cognitive Assessment (MoCA) score $>$ 26. We measured the 2D:4D digit ratio using Digital Vernier Calipers. Participants carried out the Verbal Fluency Test and Rey Auditory Verbal Learning Test (RAVLT).

\section{Results and Conclusions:}

Statistically significant differences were found in men's 2D:4D digit ratio between the three subgroups $(0.966,0.949,0.919)$. No statistically significant differences were found among women (0.981, 0.978, 0.984.). When RAVLT performance was examined statistically significant differences were found only in the subgroup 70-80 (W: 46.0- M: 35.0), whereas no differences were found in the subgroup 20-30 (W: 56.5-M: 56.3). In the VFT, women outperformed men in verbal memory, in phonemic VFT and switched more often. Men outperformed women in clustering.

Overall, we found that the most prevalent differences between men and women, both in digit ratio and in cognition, have been found in the 70-80 y.o. group. Gender differences have had a moderating tendency amongst the $2^{\text {nd }}$ and, especially the $1^{\text {st }}$ age group, with the latter showing a negative relationship regarding differences between genders. It seems that, through generations, men are exposed to less androgens (according to the 2D:4D biomarker), leading to the diminishment of differences between genders. It seems that environmental factors are continuously interacting making it challenging to investigate their effect to the feminization of the brain.

\section{D:4D digit ratio}
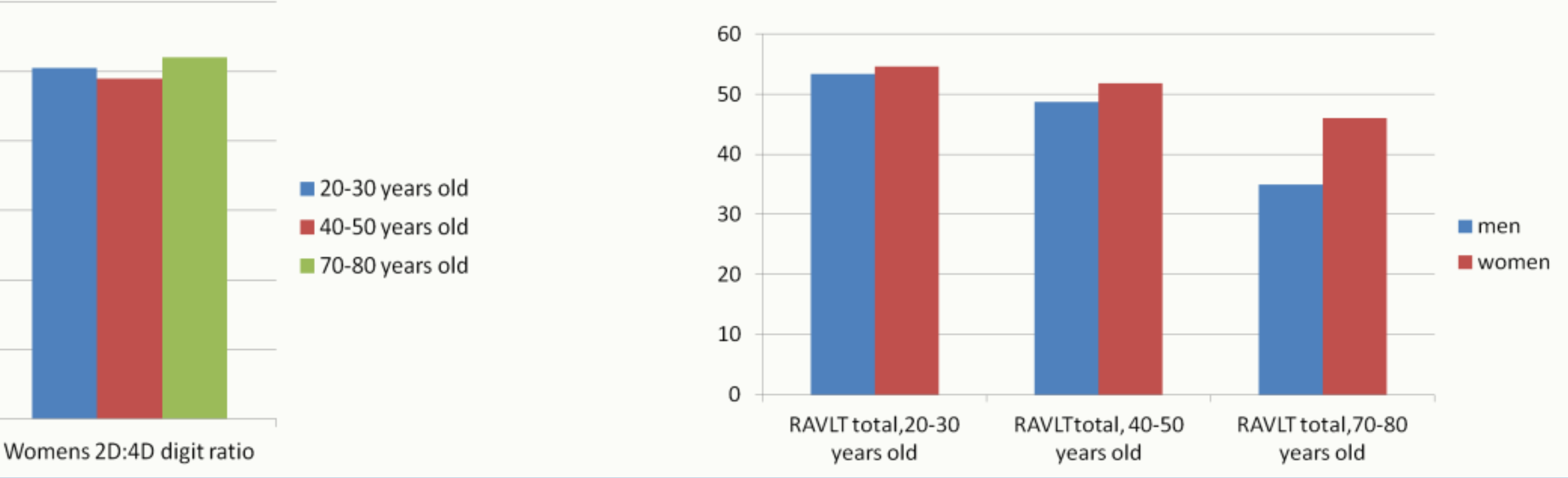